\title{
COMPOSIÇÃO FÍSICO-QUÍMICA E DE COMPOSTOS BIOATIVOS EM FRUTOS DE Bromelia antiacantha BERTOL.1
}

\author{
FERNANDA DÖRING KRUMREICH² ${ }^{2}$, ANA PAULA ANTUNES CORRÊA ${ }^{3}$, \\ SCHARLISE DIOVANELLA SCHNEIDER DA SILVA ${ }^{4}$, RUI CARLOS ZAMBIAZI ${ }^{5}$
}

RESUMO - Frutas e hortaliças, de forma geral, são fontes de vitaminas, sais minerais e fibras. Recentemente, tem sido verificada a presença de compostos bioativos, incluindo carotenoides, antocianinas e compostos fenólicos, para os quais pesquisas relatam ação antioxidante e potencial terapêutico. A Bromelia antiacantha Bertol., popularmente conhecida por banana-do-mato, caraguatá, gravatá, carauatá e croatá, ocorre naturalmente no Rio Grande do Sul-Brasil. Informações científicas quanto à composição nutricional e a atividade biológica para essa espécie ainda são restritas. Nesse sentido, o objetivo do trabalho foi determinar a composição físico-química, incluindo o teor de compostos bioativos da Bromelia antiacantha Bertol. Elevado teor de lipídios (5,07 $\pm 0,4 \%$ ), ácido ascórbico $\left(60,01 \pm 5,04 \mathrm{mg}_{\left.100 \mathrm{~g}^{-1}\right)}\right.$ e carotenoides $(162,67 \pm$ $\left.36,70 \mathrm{mg} \cdot 100 \mathrm{~g}^{-1}\right)$ foram encontrados para a espécie Bromelia antiacantha Bertol., assim como baixo teor de carboidratos $(8,75 \%)$.

Termos para indexação: banana-do-mato, carotenoides, compostos fenólicos, fibras, capacidade antioxidante.

\section{PHYSICAL AND CHEMICAL COMPOSITION AND BIOACTIVE COMPOUNDS IN Bromelia antiacantha BERTOL. FRUITS}

\begin{abstract}
Fruits and vegetables in general are sources of vitamins, minerals and fibers. Recently it has been verified the presence of bioactive compounds including carotenoids, anthocyanins and phenolic compounds, for which surveys have reported antioxidant action and therapeutic potential. The Bromelia antiacantha Bertol., popularly known as banana do mato, caraguatá, gravata, carauatá and croatá occurs naturally in Rio Grande do Sul / Brazil. Scientific information on the nutritional composition and biological activity of this species is scarce. In this sense, the objective was to determine the physical and chemical composition, including the content of bioactive compounds of Bromelia antiacantha Bertol. High fat (5.07 $\pm 0.4 \%)$, ascorbic acid $\left(60.01 \pm 5.04 \mathrm{mg} .100 \mathrm{~g}^{-1}\right)$ and carotenoids $\left(162.67 \pm 36.70 \mathrm{mg} .100 \mathrm{~g}^{-1}\right)$ were found in the Bromelia antiacantha Bertol. species, as well as low-carbohydrate (8.75\%).

Index terms: Banana do mato, carotenoids, phenolic compounds, fiber, antioxidant capacity.
\end{abstract}

${ }^{1}$ (Trabalho 127-14). Recebido em: 01-04-2014. Aceito para publicação em: 15-01-2015.

${ }^{2}$ Química de Alimentos, mestranda em Ciência e Tecnologia de Alimentos, Universidade Federal de Pelotas. E-mail - nandaalimentos@ gmail.com

${ }^{3}$ Enga. de Alimentos, Dra., bolsista recém-doutor, Embrapa Clima Temperado. E-mail-apacorrea@gmail.com

${ }^{4}$ Química Industrial de Alimentos, mestranda em Nutrição, Universidade Federal de Pelotas. E-mail-scharlisediovanella@gmail.com ${ }^{5}$ Químico Industrial, PhD. Prof. Titular do Centro de Ciências Químicas, Farmacêuticas e de Alimentos, Universidade Federal de Pelotas. E-mail- zambiazi@ufpel.tche.br, Bolsista de Produtividade em Pesquisa 2 


\section{INTRODUÇÃO}

A família Bromeliaceae é conhecida principalmente por suas características ornamentais, entretanto também é constituída por espécies frutíferas, como o abacaxi (Ananas comosus) e a banana-do-mato (Bromelia antiacantha Bertol.). A Bromelia antiacantha Bertol., popularmente conhecida por banana-do-mato, caraguatá, gravatá, carauatá e croatá, ocorre naturalmente no Brasil, principalmente nos Estados do Espírito Santo, Rio de Janeiro, São Paulo, Paraná, Santa Catarina e Rio Grande do Sul (FILIPPON et al., 2011). Esta espécie, além das características ornamentais e alimentícias já citadas, também apresenta características medicinais e industriais, reunindo em uma única espécie um potencial múltiplo de aplicações (MARQUES et al., 2007), dentre os quais podemos citar a elaboração de geleias, sorvetes e remédio contra tosse.

Informações científicas quanto à composição nutricional e a atividade biológica para esta espécie ainda são restritas; no entanto, Kinupp e Barros (2008) relatam elevado teor de sais minerais, principalmente em relação ao conteúdo de $\mathrm{Ca}, \mathrm{Mg}$ e K, quando comparada a outras frutas nativas, como a goiaba-serrana (Acca sellowiana), cerejado-rio-grande (Eugenia involucrata Bertol.) e a guabiroba (Campomanesia xanthocarpa). Filippon (2009) e Zanella (2009) relataram ação expectorante, tratamento para infecções respiratórias, além de recomendações para o tratamento de asma e de bronquite para esta espécie frutífera, enquanto Santos et al. (2009) fizeram um estudo in vitro com células cancerígenas de fibroplastos de ratos (L 929) em relação à atividade biológica desta espécie e chegaram a resultados que inibiram $20 \%$ das células cancerígenas na menor dose utilizada $(0,01 \mathrm{~g} / \mathrm{mL})$. A Bromelia antiacantha Bertol., em sua composição, também apresenta compostos fitoquímicos, como compostos polifenólicos, flavonoides e taninos (ANDRIGHETTI FROHNER et al., 2005). Os taninos, por sua vez, têm sido relatados como inibidores de enzimas digestivas e, assim, podem reduzir a digestibilidade de alguns nutrientes, como proteínas e hidratos de carbono (REDDY et al., 1985).

A riqueza de nutrientes é um dos principais fatores que conduzem ao interesse crescente pelo consumo dessas frutas e de seus produtos (RUFINO et al., 2010), uma vez que estudos epidemiológicos indicam que a ingestão frequente de frutas pode reduzir os efeitos causados pelo estresse oxidativo e, consequentemente, reduzir o risco de surgimento de várias enfermidades, como as doenças cardiovasculares, numerosos tipos de câncer, AIDS e outras diretamente associadas com o processo de envelhecimento (cataratas, doença de Alzheimer e outras alterações do sistema nervoso) (JAYAPRAKASHA et al., 2007; NEUTZLING et al., 2009). Esse efeito protetor é atribuído à variedade de substâncias antioxidantes presentes nas frutas, como algumas vitaminas (vitaminas $\mathrm{C}$ e E), compostos fenólicos (flavonoides) e carotenoides ( $\beta$-caroteno) (VETRANI et al., 2012). Nesse contexto, o objetivo do trabalho foi determinar a composição físicoquímica, incluindo o teor de compostos bioativos da Bromelia antiacantha Bertol.

\section{MATERIAL E MÉTODOS}

A fruta utilizada neste estudo foi a Bromelia antiacantha Bertol. obtida de uma propriedade rural da cidade de Canguçu - RS/Brasil (latitude: $31^{\circ} 23^{\prime} 42^{\prime \prime}$ sul, longitude: $52^{\circ} 40^{\prime} 32^{\prime \prime}$ oeste e altitude: 386 metros). Os frutos foram colhidos na segunda semana do mês de maio de 2011. A temperatura média e a precipitação pluviométrica no período de 15 de abril a 15 de maio do respectivo ano foi de $16,4^{\circ} \mathrm{C}$ e $109,7 \mathrm{~mm}$, respectivamente. O estudo foi realizado a partir de $1 \mathrm{~kg}$ de frutas maduras, definida pela coloração da casca (amarela), colhidas de forma aleatória de 10 plantas de ocorrência natural.

O trabalho foi desenvolvido nas dependências dos laboratórios de Físico-Química da área de alimentos do Centro de Ciências Químicas, Farmacêuticas e de Alimentos, e no laboratório de Cromatografia do Departamento de Ciência e Tecnologia Agroindustrial (DCTA), ambos pertencentes à Universidade Federal de Pelotas (UFPEL-RS).

Todos os resultados foram expressos como valores médios \pm desvio-padrão obtidos a partir das determinações das análises realizadas em triplicata.

As avaliações foram realizadas para a polpa da fruta após a remoção das sementes. Primeiramente, as frutas foram descascadas, cortadas no sentido longitudinal e feita a remoção das sementes; após, a polpa foi triturada, homogeneizada e armazenada em porções de $200 \mathrm{mg}$ em freezer a $-18{ }^{\circ} \mathrm{C}$ até a realização das análises.

\section{Composição centesimal}

Quantificou-se o teor de umidade, cinzas, extrato etéreo, proteína bruta e fibra bruta, conforme a metodologia descrita na AOAC (1995). O teor de carboidratos foi determinado pela diferença entre a soma dos conteúdos de umidade, cinzas, proteína, lipídios e fibra de 100 (ZAMBIAZI, 2010).

Teor de açúcares e avaliações físico-químicas 
As determinações de açúcares totais (AT \%) e de açúcares redutores (AR \%) foram realizadas segundo o método de Lane- Eynon, o qual consiste na reação de redução de um volume conhecido do reagente de Fehling. O preparo da amostra consistiu na extração aquosa dos açúcares em banho-maria, seguido de filtração e titulação. Para a determinação dos açúcares totais, a amostra filtrada foi hidrolisada com solução de ácido clorídrico $1 \mathrm{~N}$, neutralizada e utilizada para posterior titulação (AOAC, 1995).

As análises físico-químicas compreenderam a determinação do $\mathrm{pH}$, medido em pHmetro digital (Digimed DM-20); acidez total titulável, por titulação com $\mathrm{NaOH} 0,1 \mathrm{~N}$, expressa em \% de ácido cítrico; e de sólidos solúveis totais (SST), expresso em ${ }^{\circ}$ Brix através de refratômetro digital (Analytikjena). Todas as determinações seguiram os métodos descritos na AOAC (1995).

Conteúdo de compostos fenólicos, carotenoides totais e de ácido ascórbico (vitamina C)

A determinação dos compostos fenólicos foi realizada de acordo com o método descrito por Swain e Hillis (1959), com pequenas adaptações. A extração dos compostos foi feita com metanol, mediante agitação de 15 em 15 minutos por 1 hora. O extrato foi separado do material sólido através de filtração. Os compostos fenólicos foram quantificados através de reação colorimétrica, utilizando o reagente de Folin-Ciocalteau. As leituras das amostras foram feitas em espectrofotômetro (modelo Ultrospec 2000) a $765 \mathrm{~nm}$, após 2 horas de reação. Os resultados foram expressos em $\mathrm{mg}$ de eq. ao ácido gálico.100 $\mathrm{g}^{-1}$ de fruta fresca, obtidos a partir de uma curvapadrão, que foi construída a partir de uma solução de ácido gálico diluída em concentrações variando de $0-100 \mathrm{mg} / \mathrm{L}$.

A determinação dos carotenoides totais foi realizada conforme o método descrito por RodriguezAmaya (1999), com algumas adaptações. A extração foi feita com acetona gelada por 10 minutos, com auxílio de um agitador magnético. O material foi filtrado em funil de buchner com papel-filtro; após, o resíduo foi lavado com o mesmo solvente até ficar incolor. O filtrado foi transferido para um funil de separação, no qual foram acrescentados $30 \mathrm{~mL}$ de éter de petróleo e em torno de $100 \mathrm{~mL}$ de água destilada. Após a separação das fases, isolou-se a fração contendo éter de petróleo. A leitura do conteúdo de carotenoides totais foi realizada em espectrofotômetro (Ultrospec 2000) a $450 \mathrm{~nm}$. Os resultados foram calculados a partir da curvapadrão de uma solução de $\beta$-caroteno diluída em concentrações variando de $0-100 \mathrm{mg} / \mathrm{L}$ e expressos em $\mathrm{mg}$ de eq. ao $\beta$-caroteno. $100 \mathrm{~g}^{-1}$ de fruta fresca.
A quantificação de ácido ascórbico (vitamina C) foi realizada através do método titulométrico de Lorenz-Steves (ZAMBIAZI, 2010), baseado na ação redutora do ácido ascórbico, fazendo uso de soluçãopadrão de iodo e tiossulfato de sódio e solução de amido como indicador. Os resultados foram expressos em mg de ácido L-áscorbico. $100 \mathrm{~g}^{-1}$ de fruta fresca.

\section{Capacidade antioxidante}

A capacidade antioxidante foi medida frente ao radical estável DPPH (2,2- difenil-1picrilhidrazila), segundo método descrito por BrandWilliams, Cuvelier e Berser (1995). Para a obtenção do extrato, seguiu-se o mesmo procedimento descrito na determinação de compostos fenólicos. A reação foi realizada com solução de DPPH $(0,044 \mathrm{~g} / \mathrm{L})$, e a absorbância foi medida a $517 \mathrm{~nm}$ após $24 \mathrm{~h}$ de reação em local protegido da luz, à temperatura ambiente (inferior a $25^{\circ} \mathrm{C}$ ). A capacidade antioxidante foi calculada através de uma curva-padrão de trolox e expressa em mg de eq. ao trolox. $100 \mathrm{~g}^{-1}$ de fruta fresca. A curva-padrão foi construída a partir de uma solução de trolox diluída em concentrações variando de 90 a $900 \mathrm{mg} / \mathrm{L}$.

\section{RESULTADOS E DISCUSSÃO}

O teor de sólidos solúveis totais e de acidez são parâmetros indicadores de sabor e do ponto de maturação dos frutos. A Bromelia antiacantha Bertol. apresentou 15,9 $\pm 0,4^{\circ}$ Brix, e 1,53 $\pm 0,06 \%$ de acidez total (Tabela 1). O teor de sólidos solúveis totais assemelha-se ao do abacaxi (11,5 a $16^{\circ}$ Brix); entretanto, a acidez desse fruto foi mais elevada, visto que os valores encontrados para o abacaxi variam de 0,52 a $1,01 \%$ de ácido cítrico (THÉ et al., 2010; BERILLI et al., 2011). A relação entre sólidos solúveis totais e acidez titulável encontrada foi de 10,4 para Bromelia antiacantha Bertol. enquanto Prado (2009) encontrou para o abacaxi 8,3. Este dado fornece um indicativo do sabor da fruta, pois relaciona a quantidade de açúcar e de ácidos presentes. Esta relação tende a aumentar durante a maturação, devido ao aumento nos teores de açúcares e à diminuição no dos ácidos.

Quanto ao $\mathrm{pH}$, o valor determinado foi de 3,57. Segundo Pereda (2005), alimentos com pH inferior a 4,5 são considerados ácidos e não permitem o desenvolvimento de Clostridum botulinum, microrganismo indicador do tratamento térmico a ser empregado em alimentos. Prado (2009), estudando diversas frutas tropicais, encontrou um $\mathrm{pH}$ de 3,65 para o abacaxi.

Quanto ao conteúdo de açúcares totais e de 
açúcares redutores, foram encontrados valores de $10,77 \pm 0,37 \%$ e de $3,30 \pm 0,13 \%$, respectivamente. Thé et al. (2010) encontraram valores inferiores aos do presente estudo para o abacaxi, tendo encontrado para açúcares totais e redutores $8,86 \%$ e 3,23\%, respectivamente.

Os resultados relacionados à composição centesimal da Bromelia antiacantha Bertol. estão apresentados na Tabela 2.

A Bromelia antiacantha Bertol. apresentou $82,63 \pm 0,21 \%$ de umidade, valor próximo ao encontrado para o abacaxi in natura, de 83,8 a $86 \%$ (BORTOLATTO; LORA, 2008; TACO, 2011).

Com relação à quantidade de cinzas, também denominada como resíduo mineral fixo, a Bromelia antiacantha Bertol. apresentou 0,93 \pm $0,06 \%$, valor duas vezes superior ao encontrado para o abacaxi, de 0,4\% (TACO, 2011). De acordo com Bortolatto e Lora (2008), os teores de cinzas variam em função da localidade onde a variedade foi plantada e da composição do solo onde crescem. É importante observar que a composição das cinzas corresponde à quantidade de substâncias minerais presentes nos alimentos (PEREDA, 2005).

Em relação ao extrato etéreo, o valor encontrado foi de 5,07 $\pm 0,4 \%(29,19 \%$ em base seca). Santos et al. (2009) encontraram valores semelhantes, de 18,2 \% para o fruto com 27,3\% de umidade $(25,03 \%$ em base seca). Geralmente, o teor de lipídios encontrados em frutas é baixo, em torno de 0,1 a 0,6 \%; com exceção de algumas frutas, como, por exemplo, o abacate, que apresenta $8,4 \%$ de lipídios (TACO, 2011). Dessa forma, pode-se inferir que a Bromelia antiacantha Bertol. é um fruto rico em lipídios. Segundo Santos et al. (2009), 64,29 $\%$ dos ácidos graxos do óleo extraído da Bromelia antiacantha Bertol. são insaturados e, dessa fração, 20,1\% é composta de ácido oleico (C18:1) e 8,28 $\%$ de ácido linolênico (C18:3). Os lipídios, além de serem fonte de energia, auxiliam na absorção das vitaminas A, D, E e K, e de acordo com a composição de ácidos graxos podem contribuir na redução do teor de LDL -colesterol e de triacilglicerois e no aumento do HDL - colesterol (PEREIRA, 2010).

Quanto ao conteúdo de proteínas, o valor encontrado foi de $0,62 \pm 0,15 \%(3,57 \%$ em base seca), inferior ao encontrado para o abacaxi in natura (1,47\%) por Bortolatto e Lora (2008) e para a Bromelia antiacantha Bertol. por Santos et al. (2009). Esse autor encontrou 8,3\% para a fruta com $27,3 \%$ de umidade (11,4\% em base seca), três vezes superior ao encontrado no presente estudo, considerando os dados em base seca.

$\mathrm{O}$ resultado obtido para o teor de fibras para a Bromelia antiacantha Bertol. foi de 2,0 $\pm 2,0 \%$, conteúdo duas a três vezes superior ao encontrado para o abacaxi, de 0,6 a 1,0 (BORTOLATTO; LORA, 2008; TACO, 2011).

Considerando o teor de carboidratos presente em frutas comumente consumidas como maçã $(15,2 \%)$, mamão $(10,4-11,6 \%)$, banana $(26,0 \%)$ e abacaxi (13, $53 \%$ ) (TACO, 2011), o conteúdo de carboidratos encontrado na Bromelia antiacantha Bertol. é inferior, sendo este de 8,75 \%.

O teor de compostos fenólicos, carotenoides, ácido ascórbico e de capacidade antioxidante da Bromelia antiacantha Bertol., consta na Tabela 3.

$\mathrm{O}$ teor de compostos fenólicos encontrado para a Bromelia antiacantha Bertol. foi de 70,73 \pm 4,34 mg.100 g-1, valor este superior ao encontrado

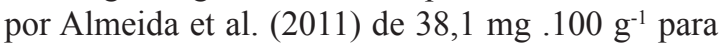
o abacaxi. Este teor é inferior quando comparado com o estudo de Rufino et al. (2010), que avaliaram os teores de compostos fenólicos de 18 espécies frutíferas nativas do Brasil e relataram conteúdos de $90,4 \mathrm{mg} .100 \mathrm{~g}^{-1}$ no umbu, a $1.176 \mathrm{mg} .100 \mathrm{~g}^{-1}$ no camu-camu.

O teor de carotenoides encontrado para a Bromelia antiacantha Bertol. foi de 162,67 \pm $36,70 \mathrm{mg} .100 \mathrm{~g}^{-1}$, valor superior ao encontrado em abacaxis, de 0,14 mg.100 g-1 (RAMOS et al., 2008) e de 0,14 a $0,78 \mathrm{mg} .100 \mathrm{~g}^{-1}$ (VIANA et al., 2013). Segundo Rodriguez-Amaya et al. (2008), para que um alimento seja considerado fonte de carotenoides, é necessário que ele possua, no mínimo, $20 \mu \mathrm{g} \cdot \mathrm{g}^{-1}$ (2 mg.100 g ${ }^{-1}$ ). Logo, podemos dizer que a Bromelia antiacantha Bertol. pode ser considerada uma fonte rica desse composto.

Quanto ao teor de ácido ascórbico (vitamina C), o valor encontrado para a Bromelia antiacantha Bertol. foi de $60,01 \pm 5,04 \mathrm{mg} .100 \mathrm{~g}^{-1}$, duas vezes superior ao encontrado para o abacaxi (34,6 mg .100 $\left.\mathrm{g}^{-1}\right)$, conforme Taco (2011). Segundo a portaria $\mathrm{n}^{\circ}$ 27, de 13 de janeiro de 1998, da Anvisa, o consumo diário recomendado de vitamina C é de $60 \mathrm{mg} / \mathrm{dia}$ (BRASIL, 1998). Conforme os resultados obtidos neste trabalho, a Bromelia antiacantha Bertol. pode ser considerada fonte de vitamina $\mathrm{C}$.

A capacidade antioxidante da Bromelia antiacantha Bertol. foi de 178,56 $\pm 2.65 \mathrm{mg}$ eq. ao trolox.100 $\mathrm{g}^{-1}$ frente ao radical DPPH, considerando que o extrato para a quantificação da atividade antioxidante foi feita em meio alcoólico (hidrofílico), pode-se inferir que o valor encontrado se refere principalmente à presença de vitamina $\mathrm{C}$ e dos compostos fenólicos presentes na amostra. Manetti et al. (2010) também observaram baixo percentual de inibição do radical DPPH para o 
extrato alcoólico desse mesmo fruto, realizando o mesmo procedimento de obtenção do extrato. Considerando que o teor de carotenoides presente na Bromelia antiacantha Bertol. é elevado, e que esses compostos também possuem potencial antioxidante, a capacidade antioxidante total para esse fruto pode ser maior quando determinado por outras metodologias.

TABELA 1- Características físico-químicas da Bromelia antiacantha Bertol.

\begin{tabular}{lccl}
\hline \multicolumn{1}{c}{ Determinações (\%) } & $\begin{array}{c}\text { Bromelia antiacantha } \\
\text { Bertol. }\end{array}$ & Abacaxi & Referências \\
\hline Sólidos solúveis totais $\left({ }^{\circ}\right.$ Brix) & $15,92 \pm 0,38$ & 11,5 a 16 (THÉ et al., 2010; BERILLI et al., 2011) \\
Acidez total(mg ác. cítrico.100 $\left.{ }^{-1}\right)$ & $1,53 \pm 0,06$ & 0,52 a 1,01 (THÉ et al., 2010; BERILLI et al., 2011) \\
Razão (SST/AT) & 10,4 & 8,3 & (PRADO, 2009) \\
pH & $3,57 \pm 0,05$ & 3,65 & (PRADO, 2009) \\
Açúcares totais $(\%)$ & $10,77 \pm 0,37$ & 8,86 & (THÉ et al.,2010) \\
Açúcares redutores $(\%)$ & $3,3 \pm 0,13$ & 3,23 & (THÉ et al.,2010) \\
\hline
\end{tabular}

Resultados expressos como média \pm desvio-padrão.

TABELA 2- Composição centesimal da Bromelia antiacantha Bertol.

\begin{tabular}{cccc}
\hline Determinações (\%) & $\begin{array}{c}\text { Bromelia antiacantha } \\
\text { Bertol. }\end{array}$ & Abacaxi & Referências \\
\hline Umidade & $82,63 \pm 0,21$ & 83,8 a 86 & (TACO, 2011; BORTOLATTO; LORA, 2008) \\
Cinzas & $0,93 \pm 0,06$ & 0,4 & (TACO, 2011; BORTOLATTO; LORA, 2008) \\
Extrato etéreo & $5,07 \pm 0,40$ & 18,2 & (SANTOS et al., 2009) \\
Proteínas & $0,62 \pm 0,15$ & 1,47 a 8,3 & (BORTOLATTO; LORA, 2008; SANTOS et al., 2009) \\
Fibras & $2,00 \pm 2,0$ & 0,6 a 1 & (BORTOLATTO; LORA, 2008; TACO, 2011). \\
Carboidratos* & 8,75 & 13,53 & (TACO, 2011) \\
\hline
\end{tabular}

Resultados expressos como média \pm desvio-padrão. *Carboidratos $=(100-$ soma dos macroelementos e do conteúdo de água $)$.

TABELA 3-Compostos fenólicos, carotenoides totais, ácido ascórbico e atividade antioxidante da Bromelia antiacantha Bertol.

\begin{tabular}{|c|c|c|c|}
\hline Determinações (\%) & Bromelia antiacantha Bertol. & Abacaxi & Referências \\
\hline $\begin{array}{l}\text { Atividade Antioxidante } \\
\left(\mathrm{mg} \text { eq. ao trolox. } 100 \mathrm{~g}^{-1}\right)\end{array}$ & $178,56 \pm 2,65$ & $*$ & $*$ \\
\hline $\begin{array}{c}\text { Compostos fenólicos } \\
\text { (mg eq. ao ác. gálico. } 100 \mathrm{~g}^{-1} \text { ) }\end{array}$ & $70,73 \pm 4,34$ & 38,1 & (ALMEIDA et al., \\
\hline $\begin{array}{l}\text { Carotenoides totais } \\
\text { (mg.eq. ao } \beta \text {-caroteno. } 100 \mathrm{~g}^{-1} \text { ) }\end{array}$ & $162,67 \pm 36,70$ & 0,14 a 0,78 & $\begin{array}{c}\text { 2011) } \\
\text { (VIANA et al., 2013) }\end{array}$ \\
\hline $\begin{array}{c}\text { Vitamina C } \\
\text { (mg.de ác. L-ascórbico.100 g-1) }\end{array}$ & $60,01 \pm 5,04$ & 34,6 & (TACO, 2011) \\
\hline
\end{tabular}

Resultados expressos como média \pm desvio-padrão. * Referência não encontrada.

\section{CONCLUSÃO}

A Bromelia antiacantha Bertol. é uma fruta rica em lipídios, ácido ascórbico e carotenoides, além de apresentar baixo conteúdo de carboidratos.

\section{REFERÊNCIAS}

ALMEIDA, M. M. B.; SOUSA, P. H. M.; ARRIAGA, A. M. C.; PRADO, G. M.; MAGALHÃES, C. E. C.; MAIA, G. A.; LEMOS, T. L. G. Bioactive compounds and antioxidant activity of fresh exotic fruits from northeastern Brazil. Food Research International, Essex, v. 44, n. 7, p. 2155-2159, 2011. 
ANDRIGHETTI FROHNER, C. R.; SINCERO, T. C. M.; DA SILVA, A. C.; SAVI, L. A.; GAIDO, C. M.; BETTEGA, J. M. R.; MANCINI, M.; DE ALMEIDA, M. T. R.; BARBOSA, R. A.; FARIAS, M. R.; BARARDI, C. R. M.; SIMÕES, C. O. M. Antiviral evaluation of plants from Brazilian Atlantic Tropical Forest. Fitoterapia, Amsterdam, v.76, n. 3-4, p. 374-378, 2005.

AOAC. Official methods of analysis of the Association of Official Analitycal Chemists. $12^{\text {th }}$ ed. Washington: Horwitz W, 1995.

BERILLI, S. S.; ALMEIDA, S. B.; CARVALHO, A. J. C.; FREITAS, S. J.; BERILLI, A. P. C. G.; SANTOS, P. C. Avaliação sensorial dos frutos de cultivares de abacaxi para consumo in natura. Revista Brasileira de Fruticultura, Jaboticabal, p. 592-598, 2011. Volume Especial .

BORTOLATTO , J.; LO RA, J. Avaliação dacomposição centesimal do abacaxi (Ananas comosus (L.) Merril) liofilizado e in natura. Revista de Pesquisa e Extensão em Saúde, América do Norte,v.4, n.1, 2008.

BRAND-WILLIAMS, W.; CUVELIER, M. E.; BERSET, C. Use of a free radical method to evaluate antioxidant activity. Lebensmittel-Wissenschaft und Technologie, London, v. 28, p. 25-30, 1995.

BRASIL. Portaria $n^{\circ} 27$, de 13 de janeiro de 1998/ANVISA. Regulamento técnico referente à informação nutricional complementar. Diário Oficial da União, Poder Executivo, Brasília, DF, 16 jan. 1998.

FILIPPON, S. Aspectos da demografia, fenologia e uso tradicional do Caraguatá (Bromelia antiacantha Bertol.) no Planalto Norte Catarinense. 2009. 104 f. Dissertação (Mestrado em Recursos Genéticos Vegetais) - Universidade Federal de Santa Catarina, Florianópolis, 2009.
FILIPPON, S.; DA SILVA, C.V.; DUARTE, A. S., BIAVATTI, SANTOS, D. S., DOS REIS, M. S. Bromelia antiacantha banana do mato. In: CORADIN, L.; SIMINSKI, A.; REIS, A. Espécies nativas da flora brasileira de valor econômico atual ou potencial: plantas para o futuro - Região Sul. Brasília: Ministério do Meio Ambiente, p. 568577, 2011.

JAYAPRAKASHA, G. K.; MANDADI, K. K.; POULOSE, S. M.; JADEGOUD, Y.; NAGANA GOWDA, G. A.; PATIL, B. S. Inhibition of colon cancer growth and antioxidant activity of bioactive compounds from Poncirus trifoliate (L.). Bioorganic \& Medicinal Chemistry, Oxford, v. 15, p. 49234932, 2007.

KINUPP, V. F.; BARROS, I. B. I. de. Teores de proteína e minerais de espécies nativas, potenciais hortaliças e frutas. Ciência e Tecnologia de Alimentos, Campinas, v. 28, n.4, p.846-857, 2008.

MANETTI, L.M.; TURRA A. F.; TAKEMURA, O. S.; SVIDZINSKI, T.I.E.;LAVERDE, J.A. Avaliação das atividades antimicrobiana, citotóxica, moluscicida e antioxidante de Bromelia antiacantha Bertol. (Bromeliaceae). Revista Brasileira de Plantas Medicinais, Botucatu, v.12, n.4, p. 406413, 2010.

MARQUES, G.; GUTIÉRREZ, A.; DEL RIO, J.C. Chemical characterization of lignin and lipophilic fractions from leaf fibers of curaua. Journal of Agricultural and Food Chemistry, Easton, v.55, n.4, p.1327-36, 2007.

NEUTZLING, M. B.; ROMBALDI, A. J.; AZEVEDO, M. R.; HALLAL, P.C. Fatores associados ao consumo de frutas, legumes e verduras em adultos de uma cidade no Sul do Brasil. Caderno de Saúde Pública, Rio de Janeiro, v.25, n.11, p.23652374, 2009.

PEREDA, J.A.O. Tecnologia de alimentos. componentes dos alimentos e processos. Porto Alegre: Artmed, v.1, p. 294, 2005. 
PEREIRA, C. M. O papel das gorduras saturadas e insaturadas na doença cardiovascular. Revista Fatores de Risco, Campo Grande, n. 16, p. 66-71, 2010 .

PRADO, E. Composição fenólica e atividade antioxidante de frutas tropicais. 2009. $106 \mathrm{f}$. Dissertação (Mestrado em Ciência e Tecnologia de Alimentos) - Escola Superior de Agricultura "Luiz de Queiroz”, Universidade de São Paulo, Piracicaba, 2009.

RAMOS. A. M; QUINTERO, A. C. F.; FARAONI, A. S.; SOARES, N. F. F.; PEREIRA, J. A. M. Efeito do tipo de embalagem e do tempo de armazenamento nas qualidades físico-química e microbiológica de abacaxi desidratado. Alimentos e Nutrição, Araraquara, v.19, n.3, p. 259-269, 2008.

REDDY, N. R.; PIERSON, M. D.; SATHE, S. K.; SALUNKHE, D. K. Taninos de feijão - uma avaliação das implicações nutricionais. Journal of the American Oil Chemists Society, Champaign, v. 62 , p. 541-549, 1985.

RODRIGUEZ-AMAYA, D. B. A Guide to carotenoid analysis in foods. Washington: International Life Sciences Institute, 1999. 64p.

RODRIGUEZ-AMAYA, D. B.; KIMURA, M.; GODOY, H. T.; AMAYAFARFAN, J. Updated Brazilian on food carotenoids: Factors affecting carotenoid composition. Journal of Food Composition and Analysis, San Diego, v. 21, p. 445-463, 2008.

RUFINO, M. S. M. , ALVES, R. E.; BRITO, E. S.; PÉREZ-JIMÉNEZ, J.; SAURA-CALIXTO, F.; MANCINI-FILHO, J. Bioactive compounds and antioxidant capacities of 18 non-traditional tropical fruits from Brazil. Food Chemistry, Barking, v.121, p.996-1002, 2010.

SANTOS, V. N. C.; FREITAS, R. A. DE; DESCHAMPS, F. C.; BIAVATTI, M. W. Ripe fruits of Bromelia antiacantha: investigations on the chemical and bioactivity profile. Revista Brasileira de Farmacognosia, João Pessoa, v. 19, n. 2, p. 358$365,2009$.
SWAIN, T.; HILLIS, W. E. The phenolic constituents of Prunus domestica L. The quantitative analysis of phenolic constituents. Journal of Science and Food Agriculture, Washington, v.10, p.63-68, 1959.

TACO. Tabela brasileira de composição de alimentos. 4.ed. Campinas: Nepa-Unicamp, 2011. $161 \mathrm{p}$.

THÉ, P.M.P.; NUNES, R.P de.; MOREIRA DA SILVA, L.I.M.; ARAÚJO, B.M de. Características físicas, físico-químicas, químicas e atividade enzimática de abacaxi cv. smooth cayenne recém colhido. Alimentos e Nutrição, Araraquara, v. 21, n. 2, p. 273-281, 2010.

VETRANI, C.; COSTABILE, G.; DI MARINO, L.; RIVELLESE, A. A. Nutrition and oxidative stress: a systematic review of human studies. International Journal of Food Sciences and Nutrition, Basingstoke, v.64, n.3, p.312-26, 2012.

VIANA, E. S.; REIS, R. C.; JESUS, J. L.; JUNGHANS, D. T.; SOUZA, F. V. D. Caracterização físico-química de novos híbridos de abacaxi resistentes à fusariose. Ciência Rural, Santa Maria, v. 43, n.7, p.1155-1161, 2013.

ZAMBIAZI, R. C. Análise físico-química de alimentos. Pelotas: Universitária, 2010. p.202.

ZANELLA, C. Caracterização genética, morfológica e fitoquímica de populações de Bromelia antiacantha (Bertol.) do Rio Grande do Sul. 2009. 98 f. Dissertação (Mestrado em Genética e Biologia Molecular) - Universidade Federal do Rio Grande do Sul, Porto Alegre, 2009. 